\title{
Faraday Waves in Cold-Atom Systems with Two- and Three-Body Interactions
}

Received: 1 December 2016 / Accepted: 23 December 2016 / Published online: 20 January 2017

(C) Springer-Verlag Wien 2017

\begin{abstract}
We report an investigation on Bose-Einstein condensates with two-body (cubic) and three-body (quintic) interactions in the corresponding nonlinear Schrödinger equation, considering $s$-wave two-body scattering length $a_{s}$ periodically varying in time. For the quintic interacting term, the dependence on $a_{s}$ was considered within two models, being quadratic or quartic. It was shown that parametric instabilities can lead to th e generation of Faraday wave resonances in this system, with wavelengths depending on the background scattering length, as well as on the corresponding modulation parameters. A few sample results are shown here for repulsive $a_{s}$, in case of quadratic and quartic three-body interactions. The effect of dissipation is also verified on the amplitude of the resonances. Analytical predictions for the resonance positions are confirmed by our numerical simulations.
\end{abstract}

\section{Introduction}

First described by Faraday in 1831 as nonlinear standing waves of a fluid on the surface of a vessel vibrating vertically [1], the Faraday waves (FW) are governed by parametric resonance equations, as waves oscillating at half the driving frequency. In the context of Bose-Einstein condensates (BEC), the interest on FW can be traced from a theoretical investigation reported in [2], with corresponding experimental observation of this

This article belongs to the Topical Collection "The 23rd European Conference on Few-Body Problems in Physics".

Presented at the 23rd European Conference on Few-Body Problems in Physics, Aarhus, Denmark, August 2016.

L. Tomio $(\varangle)$

Universidade Estadual Paulista (UNESP), Instituto de FíSica Teórica (IFT), São Paulo 01140-070, Brazil

E-mail: tomio@ift.unesp.br

L. Tomio

CCNH, Universidade Federal Do ABC, Santo André 09210-170, Brazil

L. Tomio

Instituto Tecnológico de Aeronáutica, DCTA, São José Dos Campos 12228-900, Brazil

A. Gammal

Instituto de FíSica, Universidade de São Paulo, São Paulo 05508-090, Brazil

F. K. Abdullaev

Department of Physics, Kulliyyah of Science, IIUM, 25200 Kuantan, Malaysia

F. K. Abdullaev

CCNH, Universidade Federal Do ABC, Santo André 09210-170, Brazil 
kind of patterns [3], which can be achieved by periodic modulations of the two-body atomic scattering length $a_{s}(t)$. Our motivation in studying FW in cold-atom systems is due to the fact that both two- and three-body interactions are affected by the modulation of $a_{s}(t)$, with the corresponding predictions being possible to be experimentally verified. In the present communication, by following a more detailed investigation presented in Ref. [4], we reproduce the basic expressions with a few results for repulsive $a_{s}$. Within our approach, we have a condensate with atoms of mass $m$, where the nonlinear cubic and quintic terms are functions of time [as dependent on $a_{s}(t)$ ], respectively, given by $\Gamma(t)$ and $G(t)$. As usual, with an appropriate external timeindependent trap potential, having a strong transversal trap frequency $\omega_{\perp}$, we can reduce to a one-dimensional (1D) the original three-dimensional (3D) system, such that

$$
\mathrm{i} \hbar \frac{\partial \psi}{\partial t}=-\frac{\hbar^{2}}{2 m} \frac{\partial^{2} \psi}{\partial x^{2}}+V_{e x t}(x) \psi-\Gamma(t)|\psi|^{2} \psi-G(t)|\psi|^{4} \psi
$$

Here, the wave-function $\psi \equiv \psi(x, t)$ is normalized to the number of atoms $N, \Gamma(t)$ is linearly related to the two-body scattering length $a_{s}(t)$, which can be varied in time by considering Feshbach resonance techniques [5]. Correspondingly, we have the three-body strength $G(t)$, which is also related to $a_{s}(t)$, as well as induced by some external interactions acting on the condensate. More specifically, as discussed in [4], the dependence of $G(t)$ on $a_{s}(t)$ can be quadratic or quartic. Quadratic behavior of $G(t)$ on $a_{s}(t)$ can occur in a model with 1D non-polynomial Gross-Pitaevskii (GP) equation confined in a cigar-type trap [6,7]. For small $a_{s}|\psi|^{2}$, an effective quintic term can be derived with $G(t) \equiv 2 \hbar \omega_{\perp} a_{s}^{2}(t)$, where $\hbar \omega_{\perp}$ is our defined energy unit. Other models, presenting quadratic dependences of $G(t)$ on $a_{s}(t)$ can be found in Refs. [8,9]. A quartic behavior of $G(t)$ on $a_{s}(t)$ can be verified by varying $a_{s}$ through Feshbach resonance techniques [5]. As the absolute value of this observable becomes very large, one approaches the unitary limit $\left(\left|a_{s}\right| \rightarrow \infty\right)$, also known as Efimov limit [10], where many three-body bound-states and resonances can be found. This behaviour will induce changes in the corresponding quintic parameter of the GP equation, such that we can have $G(t) \sim a_{s}^{4}(t)$ [11].

With the assumption that $V_{\text {ext }}(x)=0$, and dimensionless quantities, such that $t \rightarrow t / \omega_{\perp}, x \rightarrow l_{\perp} x$, and $\psi \rightarrow u / \sqrt{l_{\perp}}$ where $u \equiv u(x, t)$ and $l_{\perp} \equiv \sqrt{\hbar /\left(2 m \omega_{\perp}\right)}$, the Eq. (1) can be written as

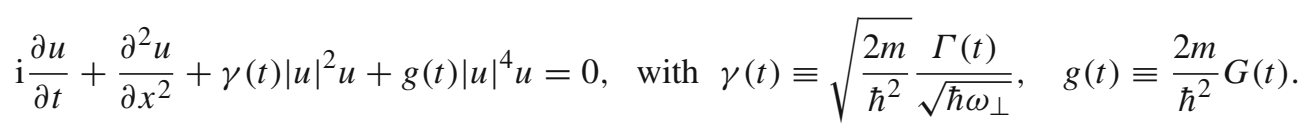

\section{Modulational Instability}

By considering modulational instability (MI) analysis of the nonlinear solution, as shown in Ref. [4], we look for a solution of the form

$$
u(x, t)=[A+\delta u(x, t)] \exp \left\{\mathrm{i}\left(A^{2} \int_{0}^{t}\left[\gamma\left(t^{\prime}\right)+A^{2} g\left(t^{\prime}\right)\right] d t^{\prime}\right)\right\}, \quad \text { with } \delta u \ll A .
$$

In order to include the inelastic three-body collisions, one should add in the formalism the term $\mathrm{i} \kappa_{3}|u|^{4} u$, replacing $g(t)$ by $g_{c}(t)=g(t)+\mathrm{i} \kappa_{3}$. By keeping only linear terms $\delta u \equiv \delta u(x, t)$, with $\delta u=v+\mathrm{i} w$, and going to the Fourier components, $(v, w)=\int e^{\mathrm{i} k x}(V, W) d k$, we have a coupled equation for $V$ and $W$. The solution in $V$, where we have the dissipative term $\sim \kappa_{3}$ together with a term $\sim \kappa_{3}^{2}$, is

$$
\frac{d^{2} V}{d t^{2}}+k^{2}\left[k^{2}-2 A(t)^{2}\left(\gamma(t)+2 A(t)^{2} g(t)\right)\right] V=-6 \kappa_{3} A(t)^{4} \frac{d V}{d t}+15\left[\kappa_{3} A(t)^{4}\right]^{2} V .
$$

The periodic modulations of the scattering length in time is given by $\gamma(t) \equiv \gamma_{0}+\gamma_{1} \cos (\omega t)$, with the threebody interaction term given by $g(t)=c\left[\gamma_{0}+\gamma_{1} \cos (\omega t)\right]^{2 n}$ ( $n=1$ for quadratic, and $n=2$ for quartic). $\gamma_{0}$ refers to the natural two-body scattering length, which can be attractive $\left(\gamma_{0}>0\right)$ or repulsive $\left(\gamma_{0}<0\right)$, with $\gamma_{1}$ being the amplitude of the periodic modulation. In Ref. [4] both the cases, with $\gamma_{0}>0$ and $\gamma_{0}<0$, were verified. However, for attractive two-body interaction, usually one needs to consider three-body repulsion to stabilise a condensate. Here, we show results only for $\gamma_{0} \leq 0$ (repulsive), with attractive quintic terms $(c>0)$, having quadratic or quartic dependence on $a_{s}$. 
Next, we derive the FW conditions for the wavenumber $k$. As the inclusion of dissipation is straightforward, for simplicity we consider the case with no dissipation $\left(\kappa_{3}=0\right)$. By considering the above periodic modulations for $\gamma(t)$ and $g(t)$, we obtain

$$
\frac{d^{2} V}{d t^{2}}+\Omega^{2}\left[1-f_{1} \cos (\omega t)-f_{2} \cos (2 \omega t)\right] V=0,
$$

where $\Omega^{2} \equiv k^{2} \Delta \equiv k^{2}\left\{k^{2}-2 A^{2}\left[\gamma_{0}+A^{2} c\left(2 \gamma_{0}^{2}+\gamma_{1}^{2}\right)\right]\right\}$, with $f_{1} \equiv\left[2 \gamma_{1} A^{2}\left(1+4 c A^{2} \gamma_{0}\right)\right] / \Delta$ and $f_{2} \equiv$ $2 c \gamma_{1}^{2} A^{4} / \Delta$. The parametric resonances will occur when matching the frequency $\omega$ with $\Omega$, in two cases: $\omega=2 \Omega(\eta \equiv 1)$ and $\omega=\Omega(\eta \equiv 2)$. The corresponding wavenumbers are given by

$$
k_{F}^{(\eta)}= \pm \sqrt{\frac{M_{ \pm}}{2}+\frac{1}{2} \sqrt{M_{ \pm}^{2}+(\eta \omega)^{2}}} \equiv \frac{2 \pi}{L_{\eta}}, \quad \text { with } M_{ \pm} \equiv 2 A^{2}\left[ \pm\left|\gamma_{0}\right|+A^{2} c\left(2 \gamma_{0}^{2}+\gamma_{1}^{2}\right)\right] .
$$

$M_{+}$is for attractive or zero two-body interactions, $\gamma_{0} \geq 0$, and $M_{-}$for the repulsive case, $\gamma_{0}<0$. In the present case, as we are analysing the case with $c>0, M_{-}$can be set to zero or negative only for repulsive interactions. Only the relevant positive sign for $k_{F}$ is being considered in our results.

\section{Results}

In order to obtain our results, we start first by considering the analytical approach derived from the modulational instability conditions. In Fig. 1 we show the behavior of the period of the FW oscillations for different values of $\omega$, as given by (6). In both panels, we have $L_{F}=L_{1}$ for $\omega=2 \Omega$ and $=L_{2}$ for $\omega=\Omega$, considering two cases with repulsive two-body interactions, using three-body interactions proportional to $a_{s}^{2}(t)$. The other parameter are such that $A=c=1$. Our search for the exact numerical solutions of the FW resonances is implemented by solving the nonlinear Eq. (2), considering the analytical predictions given by (6).

In Fig. 2, we present results for the evolution of the central density $|u(0, t)|^{2}$, when considering quadratic model for the behavior of the three-body strength $G(t)$ in terms of $a_{s}(t)$. In the upper panels, we verify the emergence of the first (left) and second (right) resonances, with no dissipation. In the lower panels, we show the effect of dissipation, by considering the value of $k$ at the resonant position. As expected, the amplitude of the resonance decreases gradually as we increase the dissipation. In the bottom-right panel we show that the effect of dissipation can be partially compensate by varying the amplitude of the time-oscillating part of the scattering length, $\gamma_{1}$. These results are reproduced from Ref. [4], where more details and results can be found. We should also observe that our analytical prediction for the resonance positions $k_{F}$ are quite well determined within our numerical search, in case of quadratic as well as quartic behavior of $G(t)$ in terms of the two-body scattering length. Verified by the time evolution results of Eq. (2), one should notice that with a small shift of $k$ from $k_{F}$, the resonant behavior vanishes completely (as indicated inside the upper panels).

In Fig. 3, we also present results for the evolution of the central density $|u(0, t)|^{2}$, when considering that $G(t) \sim\left[a_{S}(t)\right]^{4}$ (quartic model). In the left panel we verify the emergence of the first resonance at $\omega=20$ and $k=3.139$; and, in the right panel, the resonance occurs at $\omega=40$ with $k=4.45$. In both panels, the quintic
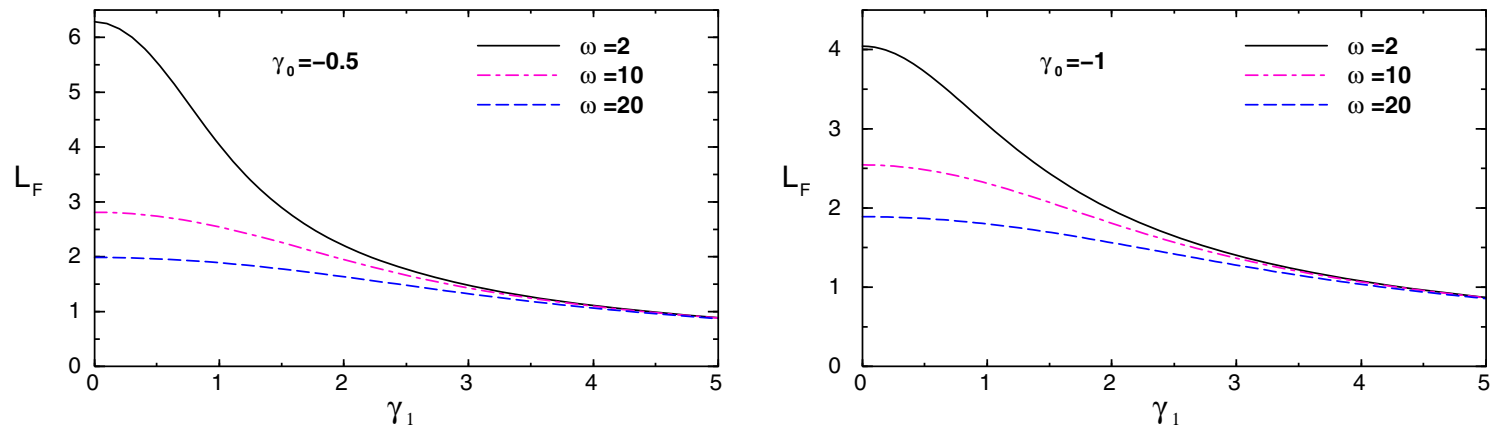

Fig. 1 Period of FW resonances for a few sets of frequencies $\omega\left(L_{F}=L_{1}\right.$ for $\omega=2 \Omega$ and $=L_{2}$ for $\left.\omega=\Omega\right)$, with two cases of repulsive two-body interactions $\left(\gamma_{0}=-0.5\right.$ and -1$)$, when the three-body interaction is proportional to $\left[a_{S}(t)\right]^{2}$. The parameters $A$ and $c$ are $=1$, with all quantities in dimensionless units. (The panels are reproduced from Ref. [4]) 

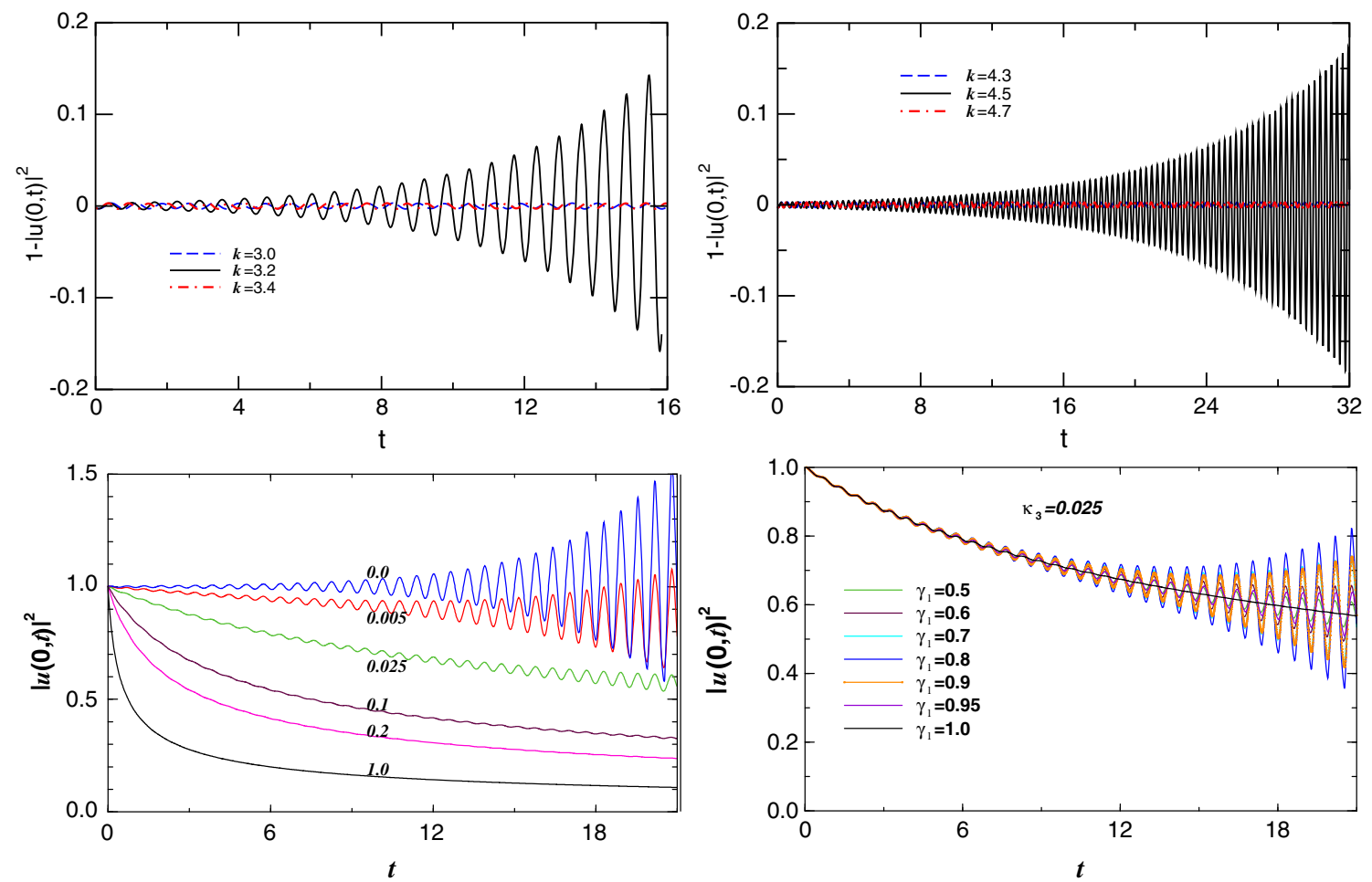

Fig. 2 For the central density $|u(0, t)|^{2}$ as function of time, in the upper-left panel we show the emergence of the first parametric resonance, for $\omega=20$, at $k=3.2$; and, in the upper-right panel, the emergence of the second parametric resonance, for $\omega=40$, at $k=4.5$. In both upper panels we have no-dissipation and the other parameters $\gamma_{0}=0, \gamma_{1}=0.5, A=1$, and $c=1$. In the lower panels we show the effect of dissipation, by considering the value of $k(=3.17)$ at the resonant position. For that, in the quintic parameter $g$ we add a dissipative term $\kappa_{3}$. In the lower-left panel, we vary $\kappa_{3}$ from zero (non-dissipative) to 1 , as indicated. In the lower-right panel, in correspondence to the lower-left panel, we show that the effect of dissipation can be compensated by varying $\gamma_{1}$. By selecting $\kappa_{3}=0.025, \gamma_{1}$ was varied from 0.5 till 1 . As verified the maximum occurs near $\gamma_{1}=0.8$. All quantities are dimensionless (Results extracted from Ref. [4])
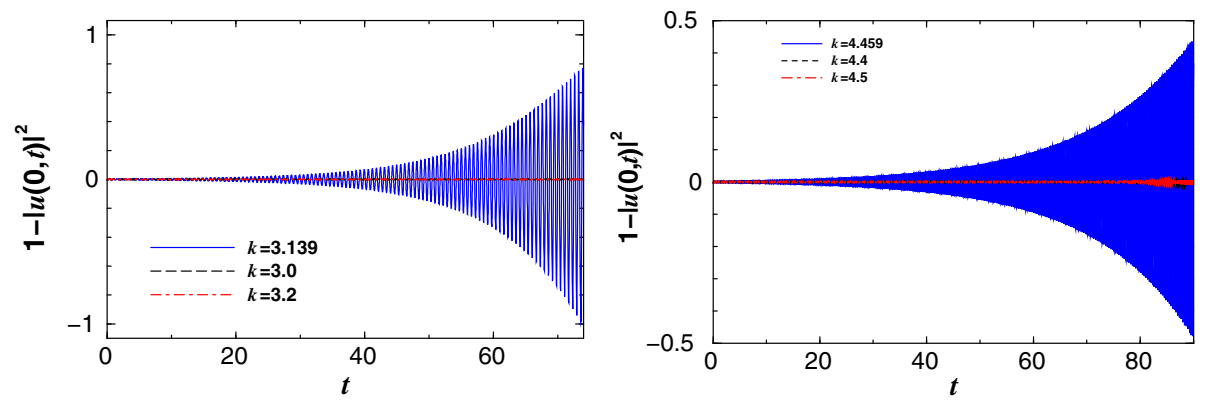

Fig. 3 Quartic case, with $g(t)>0, \gamma_{0}=-0.2, \gamma_{1}=0.2, A=1, c=+1$. The first resonance, with $\omega=20$, is found at $k=3.139$ (left panel); with the second resonance, with $\omega=40$, at $k=4.45$ (right panel). All quantities are in dimensionless units (Results extracted from Ref. [4]

term is assumed positive (attractive, $g(t)>0$ ), with the other parameters given by $\gamma_{0}=-0.2$ (repulsive two-body interaction), $\gamma_{1}=0.2, A=1, c=1$.

\section{Conclusion}

We have studied the generation of Faraday patterns in a BEC system, by engineering the time dependent two- and three-body interactions. Two models were analysed, according to the mechanism of modulation and behaviour of the three-body interaction with respect to the atomic scattering length $a_{s}$ (quadratic and quartic 
power). From our analysis, we found that the time-dependent three-body interaction excites Faraday patterns with the wave-numbers defined not only by the background scattering length and frequency of the modulations, but also by the oscillation amplitudes. Both cases, with repulsive and attractive two-body interactions, were considered in Ref. [4], where more details can be found. Only results for the repulsive case are shown in this communication.

Acknowledgements This work was partly supported by funds provided by the Brazilian agencies CAPES, CNPq and FAPESP. FKA acknowledge support from the Grant FRGS 16-014-0513 (IIUM).

\section{References}

1. M. Faraday, On a peculiar class of acoustical figures; and on certain forms assumed by a group of particles upon vibrating elastic surfaces. Philos. Trans. R. Soc. Lond. 121, 299-318 (1831)

2. K. Staliunas, S. Longhi, G.J. de Valcrcel, Faraday patterns in Bose-Einstein condensates. Phys. Rev. Lett. 89, 210406 (2002)

3. P. Engels, C. Atherton, M.A. Hoefer, Observation of Faraday waves in a Bose-Einstein condensate. Phys. Rev. Lett. 98, $095301(2007)$

4. F.Kh. Abdullaev, A. Gammal, L. Tomio, Faraday waves in Bose-Einstein condensates with engineering three-body interactions. J. Phys. B 49, 025302 (2016)

5. E. Timmermans, P. Tommasini, M. Hussein, A. Kerman, Feshbach resonances in atomic Bose-Einstein condensates. Phys. Rep. 315, 199-230 (1999)

6. L. Salasnich, A. Parola, L. Reatto, Effective wave equations for the dynamics of cigar-shaped and disk-shaped Bose condensates. Phys. Rev. A 65, 043614 (2002)

7. L. Khaykovich and B.A. Malomed, Deviation from one dimensionality in stationary properties and collisional dynamics of matter-wave solitons. Phys. Rev. A 74, 023607 (2006)

8. F. Abdullaev, A. Abdumalikov, R. Galimzyanov, Gap solitons in Bose-Einstein condensates in linear and nonlinear optical lattices. Phys. Lett. A 367, 149-155 (2007)

9. K.W. Mahmud, E. Tiesinga, P.R. Johnson, Dynamically decoupled three-body interactions with applications to interactionbased quantum metrology. Phys. Rev. A 90, 041602 (2014). (R)

10. V. Efimov, Energy levels arising from resonant two-body forces in a three-body system. Phys. Lett. B 33, 563-564 (1970)

11. A. Bulgac, Dillute quantum droplets. Phys. Rev. Lett. 89, 050402 (2002) 